Research Paper

\title{
A novel role of Cx43-composed GJIC in PDT phototoxicity: an implication of Cx43 for the enhancement of PDT efficacy
}

\author{
Deng-Pan Wu ${ }^{1,2}$, Li-Ru Bai ${ }^{3}$, Yan-Fang Lv¹, Yan Zhou ${ }^{1}$, Chun-Hui Ding1, Si-Man Yang ${ }^{4}$, Fan Zhang ${ }^{4}$, \\ Jin-Lan Huang, $1,2, \mathbb{1}$ \\ 1. Jiangsu Key Laboratory of New Drug Research and Clinical Pharmacy, Pharmacy School of Xuzhou Medical University, Xuzhou City, Jiangsu Province, \\ 221004, P.R. China \\ 2. Department of Pharmacology, Pharmacy School of Xuzhou Medical University, 221004, Xuzhou City, Jiangsu Province, P.R. China \\ 3. Department of Pharmacy,Wuxi Ninth Affiliated Hospital of Suzhou University, 214062, Wuxi City, Jiangsu Province, P.R. China \\ 4. Scientific research center of traditional Chinese medicine, Guangxi University of Chinese Medicine, Nanning, Guangxi 530200, China \\ Note: Deng-Pan Wu, Li-Ru Bai, Yan-Fang Lv contributed equally to this work.
}

$\square$ Corresponding author: Jin-Lan Huang, PhD. Jiangsu Key Laboratory of New Drug Research and Clinical Pharmacy, Pharmacy School of Xuzhou Medical University; No.209 Tongshan Road, Xuzhou City, Jiangsu Province, 221004, P.R. China; E-mail address: jinlanhuang@xzhmu.edu.cn; Tel: +86 51683262107.

(c) Ivyspring International Publisher. This is an open access article distributed under the terms of the Creative Commons Attribution (CC BY-NC) license (https://creativecommons.org/licenses/by-nc/4.0/). See http://ivyspring.com/terms for full terms and conditions.

Received: 2018.08.29; Accepted: 2018.11.28; Published: 2019.01.01

\begin{abstract}
In spite of initially promising responses, 5-year recurrence after photodynamic therapy (PDT) sustains high level and an increase in PDT effectiveness is needed. It has been demonstrated that gap junctional intercellular communication (GJIC) formed by Connexin (Cx)43 could improve the transfer of "death signal" between cells, thereby causing the enhancement of cytotoxicity of chemotherapeutics and suicide gene therapy. Nevertheless, whether Cx43-composed GJIC has an effect on PDT phototoxicity remains unknown. This study showed that Cx43-formed GJIC could improve PDT phototoxicity to tumor cells in vitro and in vivo. Specifically, Cx43-formed GJIC under the condition of high cellular density could improve PDT phototoxicity in Cx43-transfected HeLa cells and Cx43-expressing U87 glioma cells. This effect was remarkably inhibited when Cx43 was not expressed or Cx43-formed G] channels were prohibited. Additionally, the presence of Cx43-mediated GJIC could decrease the mean RTV and tumor weights of xenografts after Photofrin-PDT. The improved PDT efficacy by Cx43-composed GJIC was correlated with stress signaling pathways mediated by ROS, calcium and lipid peroxide. The present study demonstrates the presence of $\mathrm{Cx} 43$-composed GJIC improves PDT phototoxicity and suggests that therapeutic strategies designed to upregulate the expression of $\mathrm{C} \times 43$ or enhance $\mathrm{Cx} 43$-mediated GJIC function may increase the sensitivity of malignant cell to PDT, leading to the increment of PDT efficacy. Oppositely, factors that retard $\mathrm{C} \times 43$ expression or prohibit the function of $\mathrm{C} \times 43$-mediated GJIC may cause insensitivity of malignant cells to PDT, leading to PDT resistance.
\end{abstract}

Key words: Connexin 43, gap junctional intercellular communication, photodynamic therapy

\section{Introduction}

Photodynamic therapy (PDT) is an alternative cancer treatment based on the tumor-specific accumulation of a photosensitizer, followed by irradiation with specific wavelength of light, leading to cell death and the ablation of tumors via reactive oxygen species (ROS) generation [1]. PDT-mediated cancer treatment has been successfully employed in the treatments of brain, skin, lung, prostate and esophagus malignancies [2]. Photofrin, the first photosensitizer approved by the Food and Drug Administration of America in 1995, has been reported to treat esophageal cancer and other cavity tumors [3]. Initial treatment responses of PDT are infusive, nevertheless, 5-year recurrence after PDT sustain high 
level. [4]. Thus, it would be therapeutically beneficial to look for novel targets for the enhancement of PDT phototoxicity.

Gap junction (GJ) is composed of connexin (Cx) subunits. Six Cx proteins constitute a hemichannel, which docks to a counterpart in a neighboring cell for GJ formation. Gap junctional intercellular communication (GJIC) mediates the direct diffusion of molecules smaller than $1.5 \mathrm{kDa}$ from one cell to another through GJ channels. This intercellular communication has been reported to play a crucial role in cell differentiation and growth, cell proliferation and tumor suppression [5]. Additionally, GJIC can enhance cytotoxic effects of ionizing radiation and chemotherapeutics. For example, GJIC could increase the cisplatin-induced apoptosis of testicular cancer cells [6] and enhance the efficacy of ionizing radiation in human skin fibroblasts [7].

It can be inferred from the above reports that the GJIC-dependent sensitivity of cytotoxic substance relies on the "death signal" penetrating via GJ channels to neighboring unexposed cells. This is termed "bystander effect". It is noteworthy that Cx43-composed GJIC has the ability to enhance oxaliplatin cytotoxicity in colorectal cancer cell lines [8] and increase suicide gene therapy using herpesvirus vectors [9]. The findings that Cx43-mediated GIC dependent effect of cytotoxic substance indicates that the augment of Cx43-composed GJIC could improve the transfer of "death signal" between cells, leading to the enhancement of cytotoxicity of chemotherapeutics and suicide gene therapy. Nevertheless, whether Cx43-composed GJIC plays a role in Photofrin-PDT phototoxicity remains unknown.

It has been established that PDT could increase the levels of $\mathrm{ROS}, \mathrm{Ca}^{2+}$ and lipid peroxides including 4-hydroxynonenal (4-HNE) and ceramide within cells and these cytotoxic substances account for cellular injuries following PDT $[10,11]$. It is generally believed that intracellular ROS and $\mathrm{Ca}^{2+}$ can penetrate via GJ channels to surrounding neighbors [12, 13]. Additionally, since the molecular weights of 4-HNE and ceramide are smaller than $1.5 \mathrm{kDa}$, the upper limit of penetrable molecules via GJ channels, these lipid peroxides may diffuse through GJ channels. Considering the above reports, it can be reasonably hypothesized that Cx43-mediated GJIC might play a role in PDT effectiveness.

Hence, the present study was designed to explore the effect of GJIC formed by Cx43 on Photofrin-PDT-mediated phototoxicity in Cx43-transfected HeLa cells and Cx43-expressing U87 glioma cells, and its potential mechanisms. The results showed that the presence of Cx43-mediated GJIC could augment the PDT phototoxicity in transfected HeLa and U87 glioma cells and the xenografts transplanted subcutaneously with HeLa cells. This phototoxic action was related with the enhanced levels of intracellular $\mathrm{Ca}^{2+}$ and ROS, and increased amounts of ceramide by gap junctional channels. This study demonstrates that the presence of Cx43-composed GJIC improves PDT phototoxicity and suggests that therapeutic strategies designed to upregulate the expression of $\mathrm{Cx} 43$ or enhance the function of Cx43-mediated GJIC may increase the sensitivity of malignant cell to PDT, leading to the increment of PDT efficacy. Oppositely, factors that retard the expression of $\mathrm{C} \times 43$ or prohibit the function of Cx43-mediated GJIC may cause insensitivity of malignant cells to PDT, leading to PDT resistance.

\section{Material and methods}

\section{Materials}

Photofrin ${ }^{\circledR}$ (75mg/vail) was purchased from Union Med. Group Limited Company, Hong Kong, China. The reagents for cell culture were obtained from Life Technology. Doxycycline (Dox) and puromycin were obtained by Abcam. Fluo-3-Am and dichlorodihydrofluorescein diacetate (DCFH-DA) were from Beyotime, China. 4-HNE and ceramide ELISA kits were supplied by Shanghai Enzyme-linked Biotechnology Co., Ltd, China. Other reagents were all supplied by Sigma-Aldrich unless otherwise stated.

\section{Cell line and cell culture}

The HeLa cell line used in the study was stably transfected to express Cx43- formed GJ channels by controlling tetracycline operon (Tet-on) inducible gene expression system supplied by Biowit technologies (Shenzhen city, China). Cells were grown in DMEM supplemented with $10 \%$ fetal bovine serum (FBS) and $2 \mu \mathrm{g} / \mathrm{mL}$ puromycin. Cx43 expression was induced with $1 \mu \mathrm{g} / \mathrm{mL}$ Dox for $48 \mathrm{~h}$. U87 glioma cell line (ATCC) was grown in DMEM maintained with $10 \%$ FBS and $1 \%$ penicillin-streptomycin.

\section{Photofrin preparation and drug treatment}

Photofrin was freshly prepared in $5 \%$ dextrose solution for stock solution $(10 \mathrm{mg} / \mathrm{mL})$, which was then diluted to variable concentrations by DMEM when use. 18a-GA (final concentration: $10 \mu \mathrm{M}$ ) and phorbol ester (TPA, final concentration: $50 \mathrm{nM}$ ) were incubated with the cells $2 \mathrm{~h}$ before Photofrin incubation. Retinoic acid (RA, final concentration: $30 \mu \mathrm{M})$ and carbenoxolone (CBX, final concentration: $100 \mu \mathrm{M}$ ) were added $24 \mathrm{~h}$ and $10 \mathrm{~h}$ before Photofrin incubation, respectively. 


\section{In vitro photosensitivity assessment}

Cell counting kit-8 (CCK-8) and sulforhodamine $B$ (SRB) assays were used to measure the effect of Cx43-composed GJIC on PDT photosensitivity in HeLa and U87 cell line respectively according to our previous studies $[12,13]$. In brief, HeLa cells were seeded into 96-well plates at low and high cell density condition. Under high cell-density condition, cells at $3 \times 10^{4}$ cells $/ \mathrm{cm}^{2}$ were seeded, whereas, at low-density condition, cells were seeded at $3 \times 10^{3}$ cells $/ \mathrm{cm}^{2}$ into 96-well plates. At high-cell density, a single cell was allowed to contact with 3-5 others and provided a substantial opportunity of gap junctional formation. However, cells did not have chance to have a contact with each other and no GJ channels were formed at low-cell density. As for U87 cells, cells were seeded at high density. The cells were then exposed to different concentrations of Photofrin at $37^{\circ} \mathrm{C}$ for $4 \mathrm{~h}$ in the dark, and Photofrin-free medium was added before irradiation. After PDT $\left(630 \mathrm{~nm}, 20 \mathrm{~mW} / \mathrm{cm}^{2}\right.$ and 2 $\mathrm{J} / \mathrm{cm}^{2}$ ), cells were then cultured in Photofrin-free complete medium for another $24 \mathrm{~h}$. For CCK-8 assay, cells were incubated with CCK-8 solution (Dojindo Molecular Technologies, Japan) for $2 \mathrm{~h}$. For SRB assay, cells were fixed by $10 \%$ cold TCA (wt/vol) at $4^{\circ} \mathrm{C}$ for $1 \mathrm{~h}$, dyed with $0.4 \% \mathrm{SRB}(\mathrm{wt} / \mathrm{vol})$ for $30 \mathrm{~min}$ at room temperature, washed with $1 \%$ acetic acid (vol/vol) to remove the unbound dyes, and dissolve bound dyes with $10 \mathrm{mM}$ Tris base solution. The OD values were measured at 450 and $564 \mathrm{~nm}$ for CCK- 8 and SRB assay respectively, to determine cell survival using Enzyme-labeling instrument (Elx808, Bio Tek, America).

\section{In vivo photosensitivity}

For investigating the effect of Cx43-formed GJIC on photosensitivity in vivo, six-week-old BALB/c-nude mice (female) were transplanted with HeLa cells expressed Cx43 as described in our previous study [12]. In brief, the mice bearing xenografts were treated with sucrose water containing Dox $(0.2 \mathrm{mg} / \mathrm{mL}$ Dox, $5 \%$ sucrose) during the period of the experiment. The mice were intravenously injected $2.5 \mathrm{mg} / \mathrm{kg}$ Photofrin or $0.5 \%$ sterile dextrose (control), and then kept in darkness for $24 \mathrm{~h}$ followed by the irradiation at $630 \mathrm{~nm}$ at fluence rate of 75 $\mathrm{mW} / \mathrm{cm}^{2}$ for $135 \mathrm{~J} / \mathrm{cm}^{2}$. After PDT, the xenograft volume $(\mathrm{V})$ was calculated by the equation $\mathrm{V}=\mathrm{L}$ (the longest axis of xenograft) $\times \mathrm{W}$ (the axis perpendicular to $\mathrm{L})^{2} \times 0.5$. The relative tumor volume (RTV) of each xenograft was determined based on the equation $\mathrm{RTV}=$ volume of each time point/volume prior to treatment. At the end, the xenografts were excised and weighted after the mice were euthanatized. The weight inhibition rate was determined by the equation: Tumor weight inhibition rate $(\%)=(1$-mean tumor weight of treated groups/mean tumor weight of control group) $\times 100 \%$. Animal care and experimental procedures were performed in line with the document "Guidance Suggestions for Caring for Laboratory Animals" produced by the Ministry of Science and Technology of China in 2006.

\section{Haematoxylin and eosin (H\&E) staining}

The HE staining assay was performed according to our previous study [14]. In brief, the xenografts were excised and fixed in formaldehyde solution for $24 \mathrm{~h}$, then rehydrated and embedded in paraffin. Sections of tumor were prepared and stained using haematoxylin and eosin, then a light microscope was ulitised to observe histological changes after PDT under $\times 400$ magnification.

\section{Western blot assay}

The western blot assay was performed as described in our previous studies [12, 13]. Briefly, protein samples were separated and then transferred to nitrocellulose transfer membrane (Excell Bio, China). The dilution of Cx43 antibody and IRDye $800 \mathrm{CW}$ purified immunoglobulin-conjugated anti-rabbit was 1:5000 and 1:10000 respectively.

\section{"Parachute" Dye-Coupling Assay}

The assay was employed as described in our previous studies for measuring of GJ function $[12,13]$. Briefly, after donor cells were grown to $70 \%-100 \%$ confluence, cells were incubated with $5 \mu \mathrm{M}$ calcein-acetoxymethyl ester for $30 \mathrm{~min}$, the cells were trypsinized and seeded to the receiver cells (70\%-100\% confluence) at 1:150 donor/receiver ratio. Donor cells were allowed to contact with the monolayer of receiver cells for forming GJ channels for $4 \mathrm{~h}$ at $37^{\circ} \mathrm{C}$. The average amount of receiver cells containing dyes transferred by each donor cell was counted and normalized to the control group.

\section{Intracellular ROS and $\mathbf{C a}^{2+}$ detection}

Flow cytometer was utilized to determine the intracellular levels of ROS and $\mathrm{Ca}^{2+}$ based on our previous studies $[12,13]$. In brief, after cells at $70 \%-100 \%$ confluent were incubated with or without Photofrin for $4 \mathrm{~h}, 20 \mathrm{mM}$ DCFH-DA and $5 \mu \mathrm{M}$ Fluo-3-Am were added and incubated with cells for 1 $\mathrm{h}$ for ROS and $\mathrm{Ca}^{2+}$ measurement respectively. For measurement of intracellular ROS, 30 min after PDT $\left(20 \mathrm{~mW} / \mathrm{cm}^{2}\right.$ and $\left.2 \mathrm{~J} / \mathrm{cm}^{2}\right)$, cells were harvested. As for intracellular $\mathrm{Ca}^{2+}$ detection, cells were bathed in fresh $\mathrm{Ca}^{2+}$ balanced salt solution $\left(2 \mathrm{mM} \mathrm{MgCl}_{2}, 10 \mathrm{mM}\right.$ HEPES, $140 \mathrm{mM} \mathrm{NaCl}, 2.8 \mathrm{mM} \mathrm{KCl}$ and $2 \mathrm{mM} \mathrm{CaCl}_{2}$, $\mathrm{pH}$ 7.2) or $\mathrm{Ca}^{2+}$-free balanced salt solution $(140 \mathrm{mM}$ $\mathrm{NaCl}, 10 \mathrm{mM}$ HEPES and $2.8 \mathrm{mM} \mathrm{KCl}, \mathrm{pH} 7.2)$ and 
irradiated at $630 \mathrm{~nm}\left(20 \mathrm{~mW} / \mathrm{cm}^{2}\right.$ and $\left.2 \mathrm{~J} / \mathrm{cm}^{2}\right)$. After PDT, cells were washed and harvested. A flow cytometer (MACS Quantify, Germany) was utilized to measure the fluorescence intensities of ROS and $\mathrm{Ca}^{2+}$. The fluorescence intensities of $\mathrm{Ca}^{2+}$ and $\mathrm{ROS}$ of Dox-treated and -untreated cells in Photofrin-treated group were normalized to Dox-treated and -untreated cells in control group respectively.

\section{4-HNE and ceramide measurement}

ELISA assay was employed to measure the levels of 4-HNE and ceramide according to our previous studies $[12,13]$. Briefly, cells at $70 \%-100 \%$ confluence were incubated with or without $2.5 \mu \mathrm{g} / \mathrm{mL}$ Photofrin for $4 \mathrm{~h}$. $1 \mathrm{~h}$ after PDT $\left(20 \mathrm{~mW} / \mathrm{cm}^{2}\right.$ and $\left.2 \mathrm{~J} / \mathrm{cm}^{2}\right)$, cells were harvested. Then, the levels of 4-HNE and ceramide were determined using ELISA kit according to manufacturers' protocols. The levels of 4-HNE and ceramide of Dox-treated and -untreated cells in Photofrin-treated group were normalized to Dox-treated and -untreated cells in control group respectively.

\section{Statistical analysis}

The data were represented as mean \pm SD. One-way analysis of variance (ANOVA) or $t$-test was performed using SPSS software for Windows 20.0 for analyzing the variance. $P<0.05$ was considered to be statistically significant.

\section{Results}

\section{PDT phototoxicity is cell-density dependent}

For determining the role of GJIC in the survival of Cx43-transfected HeLa cells after PDT, cells were cultured in two cell-density conditions: a high-cell density where cells were allowed to form gap junctional channels, and a low-cell density in which cells did not directly contact with each other and no GJ was formed. As indicated in Figure 1, after treatment with Photofrin, cell survival was remarkably reduced in a concentration-dependent manner at both high- and low-cell density. It is noteworthy that at high-cell density, the phototoxicity of PDT was significantly augmented than that at low-cell density. In specific, the survival fraction at low density were approximately 1.5, 2.8 and 4 folds higher than that in high density when cells were treated with 2.5, 5 and $10 \mu \mathrm{g} / \mathrm{mL}$ Photofrin respectively (Figure 1). However, cell survival exhibited no significant difference when cells were treated with $1 \mu \mathrm{g} / \mathrm{mL}$ Photofrin. The above results suggest that the photocytotoxic action of PDT enhances when intercellular GJ channels are formed.

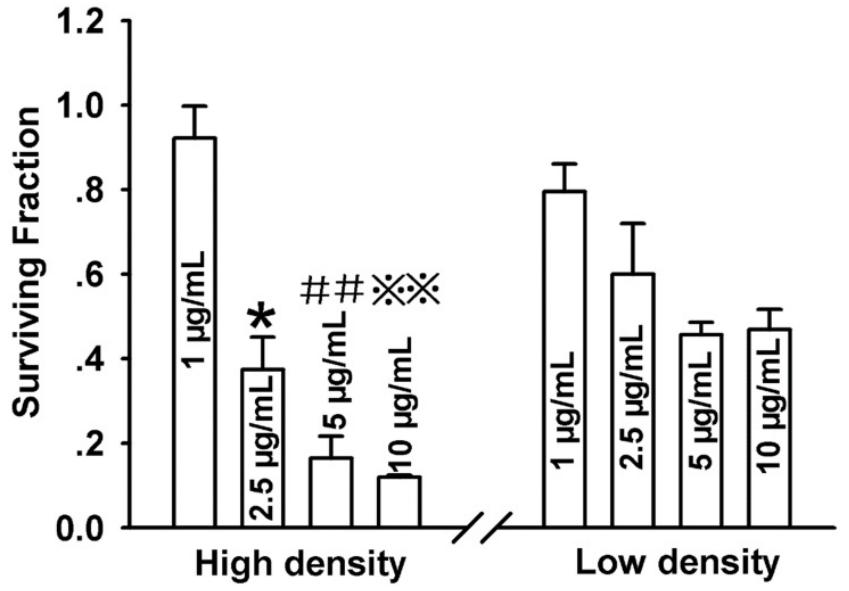

Figure 1. Phototoxicity of Photofrin-mediated PDT is cell-density dependent. CCK-8 assay was used to measure the survival of cells incubated by different concentrations of Photofrin at high-density condition $\left(3 \times 10^{4}\right.$ cells $\left./ \mathrm{cm}^{2}\right)$ or at low-density condition $\left(3 \times 10^{3}\right.$ cells $\left./ \mathrm{cm}^{2}\right)$. Data were represented as mean $\pm S D$ from 3 4 independent experiments. $t$-test was performed to analyze statistical difference between groups using SPSS 20.0. ${ }^{*} P<0.05, \ldots P<0.01$ and ${ }^{*}{ }^{*} P<0.01$ versus $2.5 \mu \mathrm{g} / \mathrm{mL}, 5 \mu \mathrm{g} / \mathrm{mL}$ and $10 \mu \mathrm{g} / \mathrm{mL}$ Photofrin-treated group at low-density, respectively.

\section{Cell-density dependence of PDT phototoxicity is mediated by Cx43-formed GJIC}

That phototoxicity of Photofrin-mediated PDT depended on cell density suggests that Cx43-composed GJIC may exhibit an effect on PDT phototoxicity. To determine the role of GJIC composed by $\mathrm{C} \times 43$ in phototoxic action of PDT, Cx43 expression and GJ function at the condition of high-cell density were manipulated by two methods: chemical inhibition by GJIC inhibitor and Dox induction of Cx43 expression. Figure 2A showed that Cx43 expression was induced by Dox-treated cells, while the expression of $\mathrm{Cx} 43$ was not induced in Dox-untreated cell. Additionally, parachute assay was performed to measure GJ function of cultured cells as described in the Materials and Methods section. In the assay, donor cells dyed with calcein were incubated with receiver cells. The function of GJ channel was assessed by the number of receiver cells labeled with calcein from donor cells. As indicated in Figure 2B, GJIC were detected in Dox-treated cells, while no GJIC was found in Dox-untreated cells. The function of GJIC was significantly decreased after Dox-treated cells were pretreated with $10 \mu \mathrm{M} 18 \mathrm{a}-\mathrm{GA}$, a GJ inhibitor verified to suppress the function of $G J$ channels (Figure 2C).

As illustrated in Figure 2D, GJ formation resulted in a substantial decrease in cell survival after treatment with $2.5 \mu \mathrm{g} / \mathrm{mL}$ Photofrin. To be specific, cell survival in Dox-treated (GJ-formed) cells were about 1.8 times lower than in Dox-untreated (GJ-unformed) cells. After Cx43-expressing cells treated with 18a-GA, cell survival was reduced 
compared to 18a-GA-untreated cells after cells were treated with $2.5 \mu \mathrm{g} / \mathrm{mL}$ Photofrin (Figure 2E). However, cell survival had no significant change between 18a-GA-treated and -untreated cells or Dox-treated and -untreated cells when cells were treated with 1, 5 and $10 \mu \mathrm{g} / \mathrm{mL}$ Photofrin (Figure 2D and E).

The above findings suggest that at medium concentration of Photofrin, under the circumstance of high-cell density where cells have changes to contact with each other and GJ is potentially formed, Cx43-composed GJIC holds a potential to augment the PDT phototoxicity. However, when $\mathrm{Cx} 43$ is not expressed or Cx43-mediated GJIC is restrained, the sensitivity of cells to PDT was substantially reduced, causing increased cell survival at high density. Collectively, these results demonstrate that the increment of PDT phototoxicity at high-cell density is mediated by Cx43-formed GJIC.

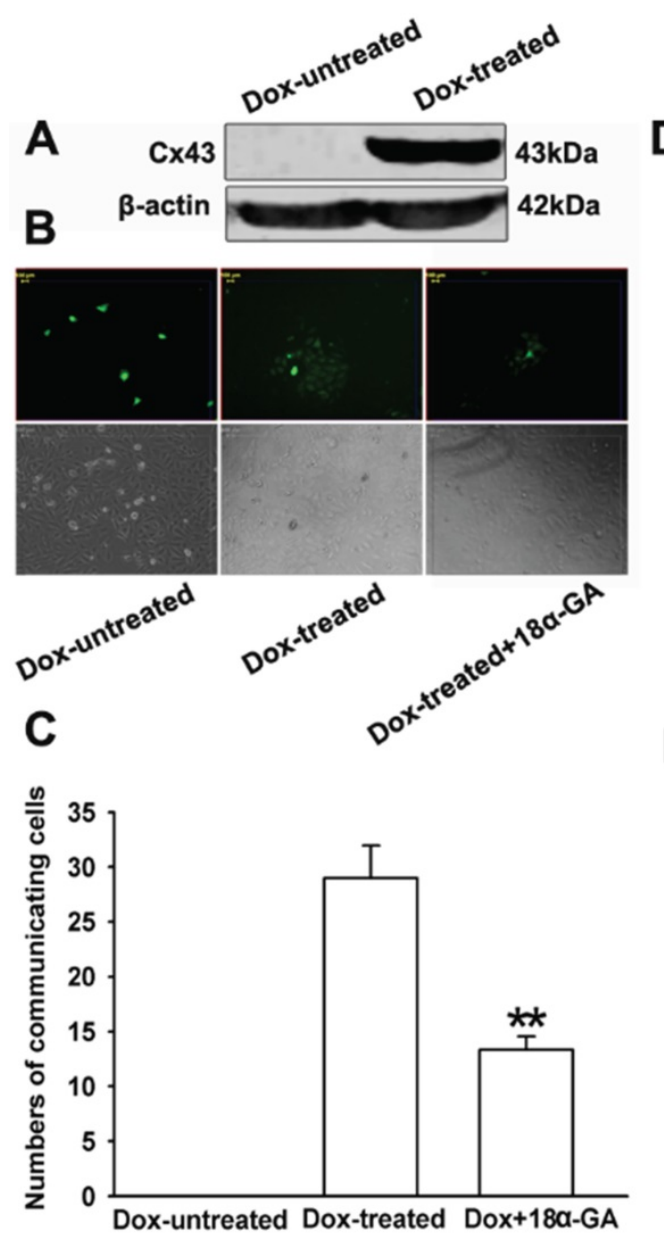

$\mathbf{E}$

\section{Increased PDT phototoxicity by Cx43-formed GJIC in U87 glioma cells}

The role of Cx43-formed GJIC in Photofrin-mediated PDT was further confirmed by seeding U87 glioma cells, a cell line expressing endogenous $\mathrm{C} \times 43$, at high-cell density. TPA and CBX, Cx43-formed GJ inhibitors, and RA, a compound being reported to enhance $\mathrm{Cx} 43$-composed GJIC, were used to manipulate the function of $\mathrm{Cx} 43$-meidated GJIC in U87 cells. Figure 3A D showed that TPA and CBX could remarkably inhibit Cx43-composed GJIC since the results of parachute assay showed that the amount of receiver cells receiving dyes from donor cells was significantly reduced in TPA- and CBX-treated groups compared to TPA- and CBX-untreated groups. In contrast, RA could substantially increase the amount of receiver cells with dyes when compared to the controls, indicating that RA enhances Cx43-composed GJIC (Figure 3E and F).
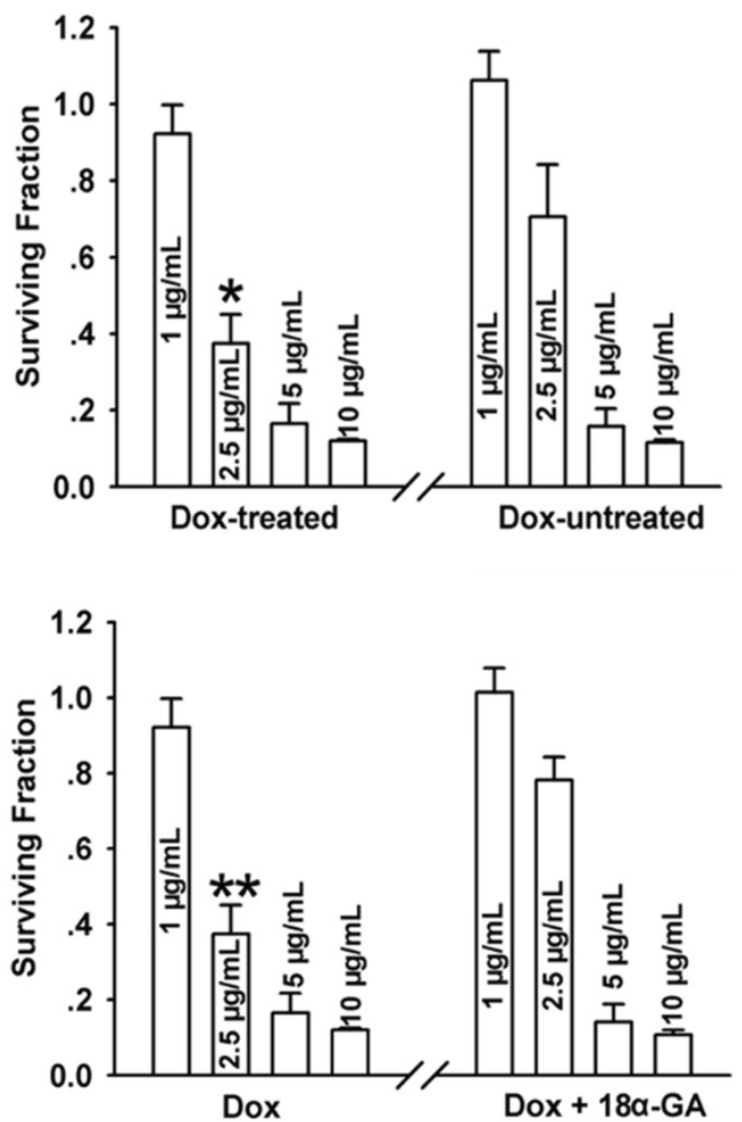

Figure 2. (A), (B) and (C): Dox induced Cx43 expression and 18a-GA inhibited $G$ J channels. (A): Western blot assay was used to detect $C \times 43$ expression. (B) and (C): "parachute" dye-coupling assay was performed to measure Gl function after cells were treated with $10 \mu M 18 \alpha-G A$. Data were represented as mean \pm SD from 3 independent experiments. $t$-test was performed to analyze statistical difference between groups using SPSS 20.0. $* * P<0.01$, versus Dox-treated group. (D) and (E): Effects of Dox and 18a-GA on the cell survival after PDT. Data were represented as mean \pm SD from 3 4 independent experiments. $t$-test was performed to analyze statistical difference between groups using SPSS 20.0. $* P<0.05, * * P<0.01$, versus Dox-untreated and Dox $+18 \alpha-G A$ treatment group, respectively. 
A

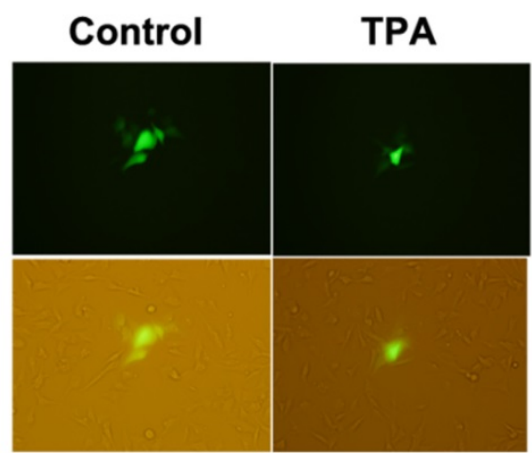

B

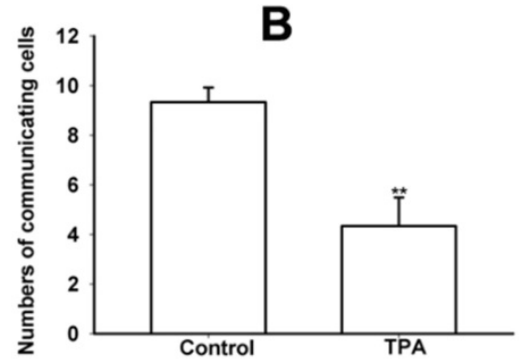

G

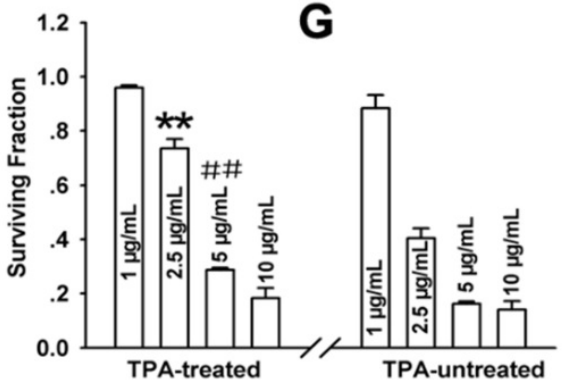

C
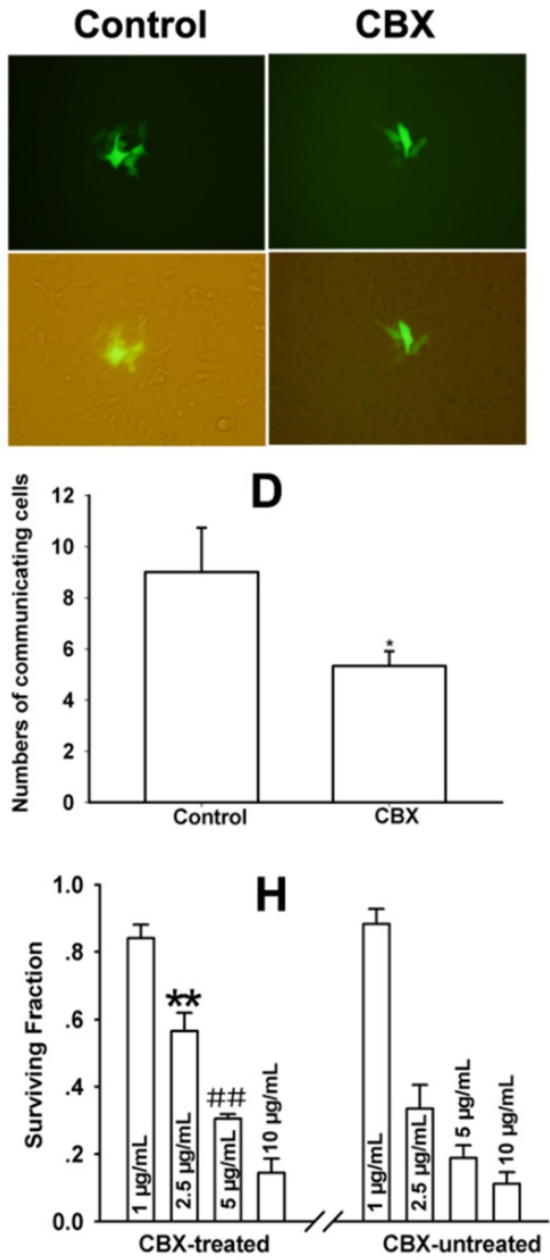

E

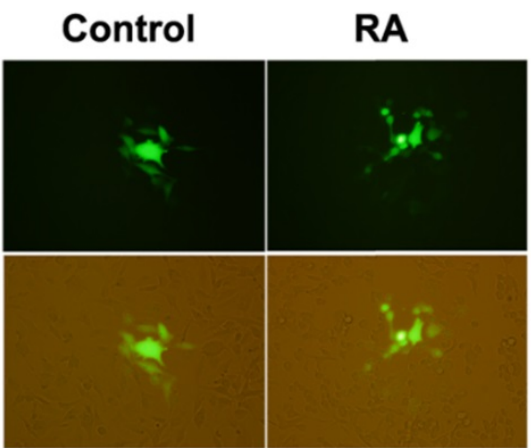

F
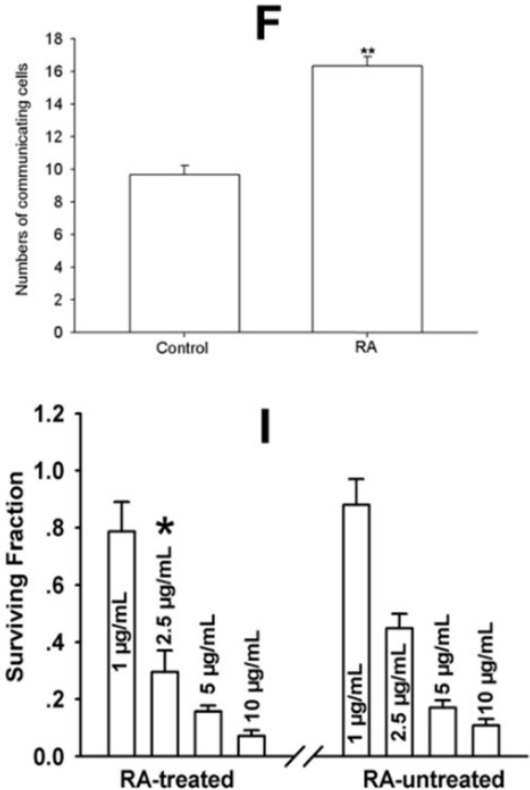

Figure 3. Cx43-formed GIIC increases phototoxicity of Photofrin-mediated PDT in U87 glioma cells. (A F) Effects of TPA, CBX and RA on Cx43-composed GJ channel in U87 glioma cells respectively. The function of GJ channel was assessed by "parachute" dye-coupling assay. Data were represented as mean \pm SD from 3 independent experiments. $t$-test was performed to analyze statistical difference between groups using SPSS 20.0 . $* P<0.05$, $* * P<0.01$ versus control group. (G I) Effects of TPA, CBX and RA on the survival of U87 cells after PDT respectively. The survival of U87 cells was evaluated by $S R B$ assay. $* P<0.05$, $* * P<0.01$, \#P<0.01versus TPA-, CBX- and RA-untreated group treated by $2.5 \mu \mathrm{g} / \mathrm{mL}$ and $5 \mu \mathrm{g} / \mathrm{mL}$ Photofrin, respectively.

The phototoxicity of Photofrin-PDT was evaluated using SRB assay. As illustrated in Figure 3G I, cell survival in TPA- and CBX-treated cells was significantly increased whereas cell survival in RA-treated cells was substantially reduced when compared to the control cells. In specific, at 2.5 and $5 \mu \mathrm{g} / \mathrm{mL}$ Photofrin, the survival fraction of TPA-treated cells was about 1.8 times higher than TPA-untreated cells, while in CBX-treated cells, survival fraction was approximately 1.5 folds higher than CBX-untreated cells (Figure $3 \mathrm{G}$ and $\mathrm{H}$ ). Whereas, the survival of RA-treated cells reduced by a fact of approximately 1.5 times than that of RA-untreated cells when cells were treated with $2.5 \mu \mathrm{g} / \mathrm{mL}$ Photofrin (Figure 3I). These results suggest that at medium Photofrin concentration, Cx43-formed GJIC has an ability to increase phototoxicity of Photofrin-mediated PDT in U87 glioma cells.

\section{Improved PDT efficacy by Cx43-formed GJIC in vivo}

For exploring the role of Cx43-composed GJIC in PDT phototoxicity in vivo, BALB/c-nude mice transplanted with Cx43-transfected HeLa cells were fed with drinking water containing with or without Dox to control the expression of $\mathrm{Cx} 43$ in tumor xenografts. Before PDT treatment, tumor xenografts randomly selected from Dox-treated and Dox-untreated group were excised for measuring Cx43 expression. As illustrated in Figure 4A, Cx43 expression in the xenografts of the mice treated with Dox was induced.

After xenografts were grown to $100-300 \mathrm{~mm}^{3}$, Photofrin or $0.5 \%$ sterile dextrose were given via tail vein. $24 \mathrm{~h}$ after the administration, the xenografts were irradiated at $630 \mathrm{~nm}$ at fluence rate of 75 $\mathrm{mW} / \mathrm{cm}^{2}$ for $135 \mathrm{~J} / \mathrm{cm}^{2}$. After irradiation, tumor 
growth was measured by monitoring tumor volume every 2 days for 10 days and the mean RTV of each xenograft was calculated. Figure 4B indicated that the tumor growth in both Dox-treated and Dox-untreated mice was remarkably prohibited after PDT. Importantly, Dox-treated xenografts exhibited a significantly decrease in the mean RTV and tumor weights when compared to Dox-untreated xenografts after Photofrin-PDT (Figure $4 \mathrm{~B} \sim \mathrm{D}$ ). The tumor weight inhibitory rates of Dox-treated and Dox-untreated group were $88.47 \%$ and $77.31 \%$ respectively (Table 1 ). The above results suggest that Cx43-composed GJIC has an ability to improve Photofrin-mediated PDT efficacy in vivo.

The effect of Cx43-composed GJIC on PDT efficacy was histologically assessed using $\mathrm{H} \& \mathrm{E}$ staining. Figure $4 \mathrm{E}$ showed that the xenograft tissues in Dox-treated and -untreated groups were filled with dense tumor cells, while tumor tissues in Photofrin-treated groups demonstrated various degrees of necrosis. Notably, A lower density of tumor cells was observed in Dox-treated group than that in Dox-untreated group after PDT (Figure 4E), indicating an enhanced phototoxicity of PDT by Cx43-composed GJIC.

Table 1. Tumor weight inhibitory rates after Photofrin-mediated PDT*

\begin{tabular}{ll}
\hline Groups & Tumor weight inhibition (\%) \\
\hline Dox-treated & 88.47 \\
Dox-untreated & 77.31 \\
\hline
\end{tabular}

*Tumor weight inhibitory rates are calculated at the end of treatment as described above.
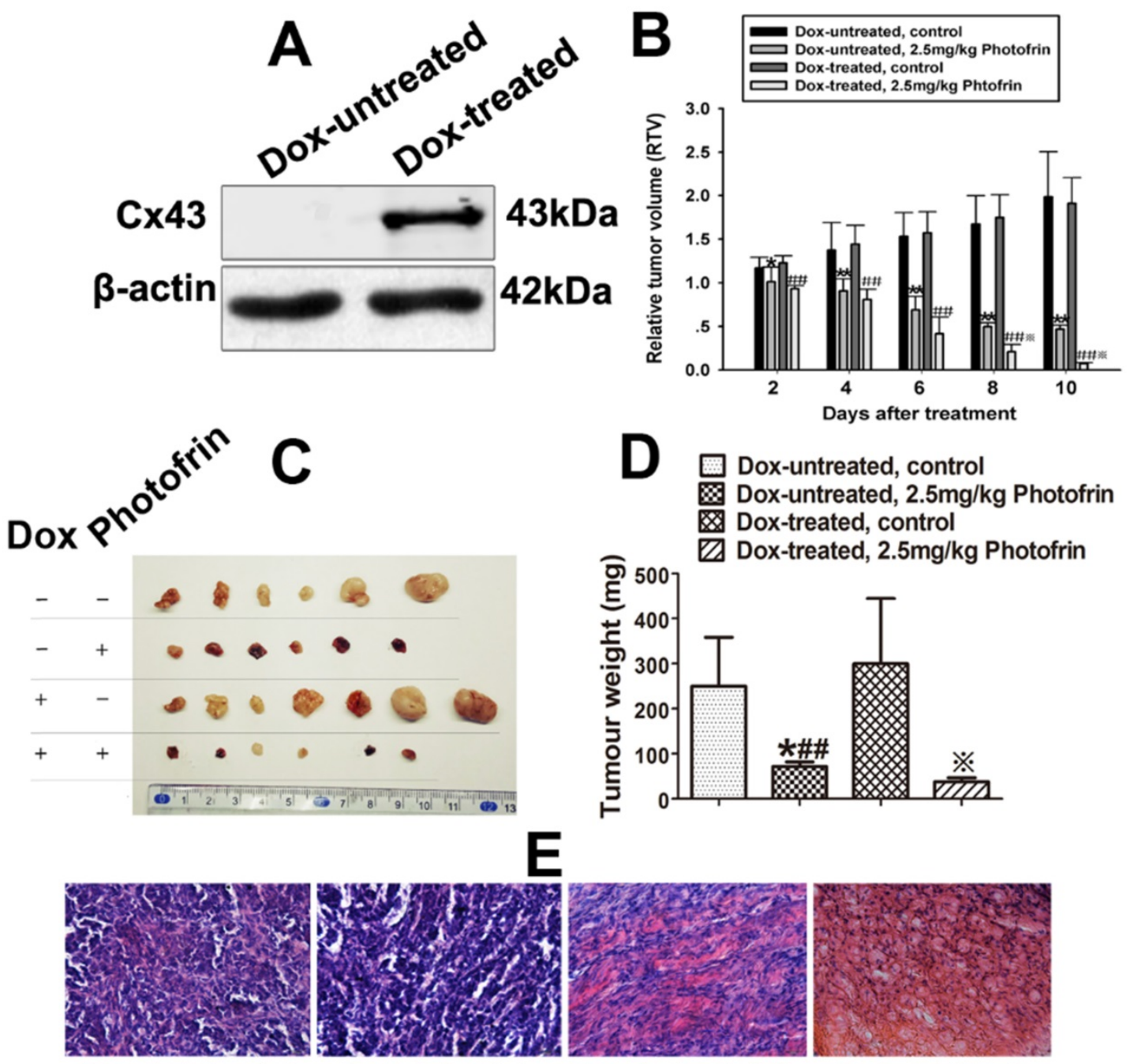

Dox-untreated, control

Dox-treated, control

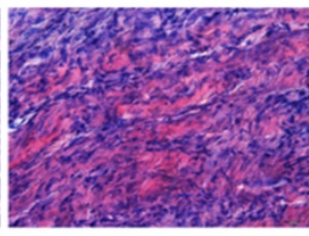

Dox-untreated, Photofrin

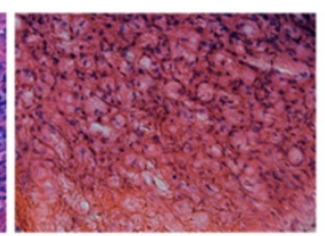

Dox-treated, Photofrin

Figure 4. Cx43-composed GJIC improves Photofrin-mediated PDT efficacy in vivo. (A) Cx43 expression induced by Dox in the xenografts was detected by Western blot; (B) Effects of Cx43-formed GJIC on the relative tumor volume (RTV) of mice after PDT; (C) and (D) Tumor weights of mice after Photofrin-mediated PDT; (E) Histological changes of xenografted tumors after PDT was assessed using H\&E staining assay. Micrographs of represented sections of tumors were observed using a light microscope under $\times 400$ magnification. For (B), (C) and (D), data represent mean \pm SD from 6 7 independent samples. One-way ANOVA was performed to analyze statistical difference between groups using SPSS 20.0. $* P<0.05$, $* * P<0.01$ versus control group (Dox-untreated); (Dox-untreated); ${ }^{*} p<0.05,{ }^{*}{ }^{*}<0.01$, versus $2.5 \mathrm{mg} / \mathrm{kg}$ Photofrin group (Dox-untreated). 

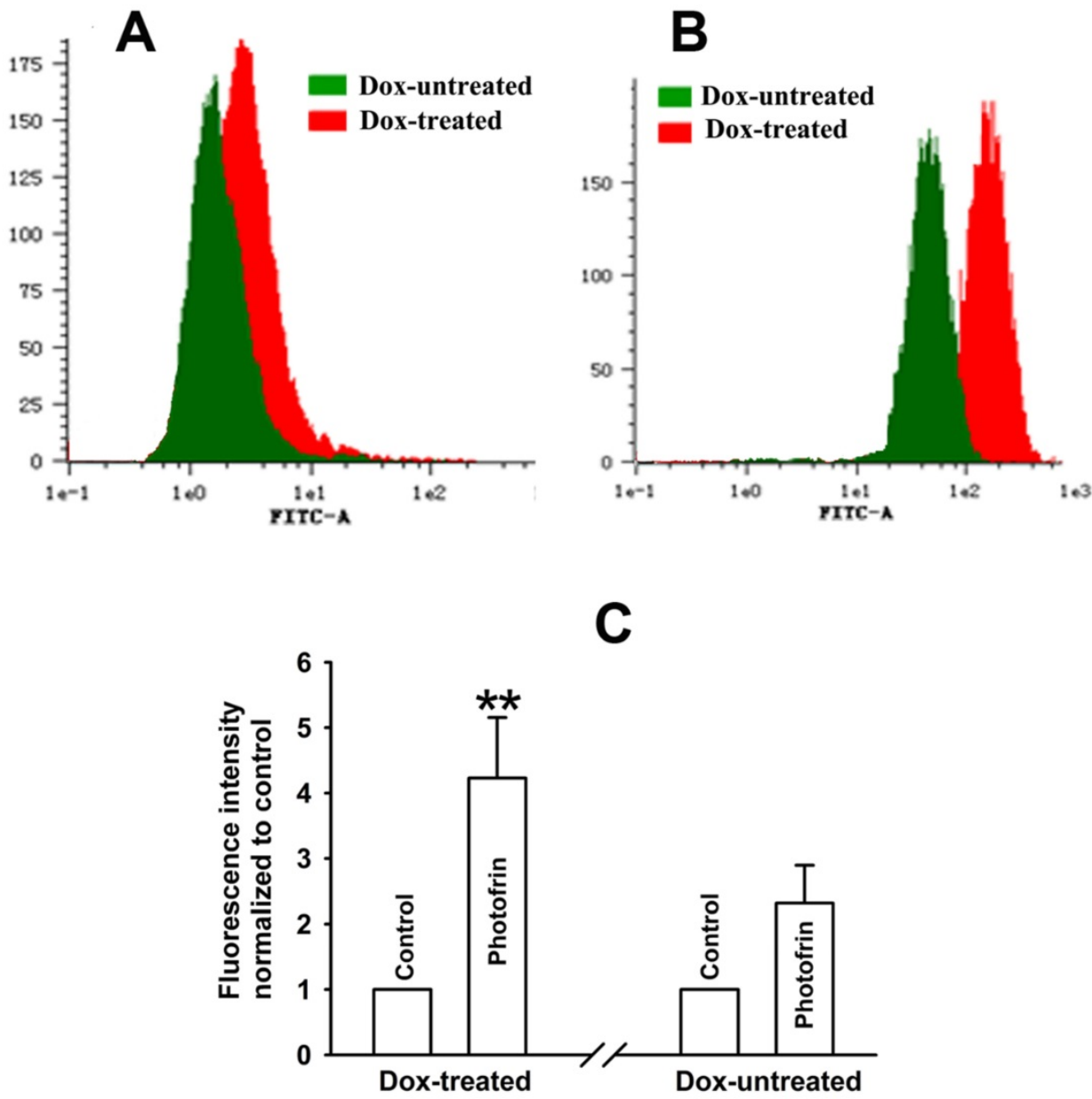

Figure 5. Enhancement of ROS production by Cx43-formed GJIC followed by PDT. The cells were treated with or without Dox, followed by incubation with DCFH-DA and irradiation with or without $2.5 \mathrm{mg} / \mathrm{mL}$ Photofrin. $30 \mathrm{~min}$ after PDT, flow cytometry was used to detect fluorescence intensity of ROS. (A): control; (B): $2.5 \mathrm{mg} / \mathrm{mL}$ Photofrin; (C): The fluorescence intensity of ROS. Data were represented as mean \pm SD from 5 independent experiments. $t$-test was performed to analyze statistical difference between groups using SPSS 20.0. ** $P<0.01$, versus Dox-untreated group.

\section{Enhanced intracellular ROS production by Cx43-formed GJIC followed by Photofrin-mediated PDT}

It has been reported that PDT induces ROS production and ROS threatens the integrity of proteins and DNA, causing cellular damage [15]. It can be assumed that ROS may play a part in Cx43-mediated GJIC-dependent augment of phototoxicity induced by Photofrin-PDT since ROS can penetrate via GJ channels. As shown in Figure $5 \mathrm{~A} \sim \mathrm{C}$, the quantities of ROS in Dox-treated cells were significantly higher than that in Dox-untreated cells. Specifically, the amount of intracellular ROS in Dox-treated cells increased by a factor of approximately 1.5 folds than that of Dox-untreated cells. The results indicate that Cx43-mediated GJIC holds a potential to augment ROS production, which may be involved in the improved phototoxicity of Photofrin-PDT by Cx43-formed GJIC.

\section{Increased extracellular $\mathrm{Ca}^{2+}$ influx and intracellular $\mathrm{Ca}^{2+}$ release by $\mathrm{Cx} 43$-formed GJIC followed by Photofrin-mediated PDT}

Reports have proven that PDT triggers the influx of $\mathrm{Ca}^{2+}$ from extracellular medium and the intracellular $\mathrm{Ca}^{2+}$ release from $\mathrm{Ca}^{2+}$ store, resulting in an increased level of intracellular $\mathrm{Ca}^{2+}$ concentration $\left(\left[\mathrm{Ca}^{2+}\right]_{\mathrm{i}}\right)$, causing apoptosis and cell death [16]. It has been confirmed that $\mathrm{Ca}^{2+}$ can transfer via GJ channels for the regulation of cellular function [17]. Thus, the presence of GJIC may facilitate $\mathrm{Ca}^{2+}$ release and/or influx. For exploring the role of Cx43-composed GJIC in $\mathrm{Ca}^{2+}$ influx after PDT, cells were illuminated in $\mathrm{Ca}^{2+}$-containing balanced salt solution after exposure to Photofrin. The results showed that in the presence of $\mathrm{Ca}^{2+}$ in extracellular medium, $\left[\mathrm{Ca}^{2+}\right]_{\mathrm{i}}$ of Cx43-expressing (Dox-treated) cells was significantly higher than that of cells not expressing Cx43 (Dox-untreated) (Figure $6 \mathrm{~A} \sim \mathrm{C})$, indicating Cx43-composed GJIC facilitates extracellular $\mathrm{Ca}^{2+}$ influx after PDT. For investigating the role of 
Cx43-composed GJIC in $\mathrm{Ca}^{2+}$ release after PDT, cells were irradiated in $\mathrm{Ca}^{2+}$-free balanced salt solution after Photofrin treatment. As shown in Figure 7D F, the level of $\left[\mathrm{Ca}^{2+}\right]_{\mathrm{i}}$ of Dox-treated (GJ-formed) cells significantly increased when compared to Dox-untreated (GJ-unformed) cells, indicating that Cx43-composed GJIC may stimulate intracellular $\mathrm{Ca}^{2+}$ release after PDT.

\section{Elevated ceramide generation by Cx43-formed GJIC followed by Photofrin-mediated PDT}

It has been reported that intracellular lipid peroxides can be accumulated after PDT [10, 11]. Among the lipid peroxides, 4-HNE and ceramide might pass through GJ channels since they have small molecular weights $(<1.5 \mathrm{kDa})$. Therefore, we hypothesized that these cytotoxic lipid peroxides might account for the enhanced PDT phototoxicity by Cx43-meidated GJIC. The results showed that ceramide generation was remarkably increased by Cx43-meidated GJIC. Specifically, the intracellular level of ceramide was significantly 2.8 times higher in Dox-treated cells than Dox-untreated cells (Figure 7B). However, there exhibited no significant difference in the level of 4-HNE between Dox-treated and Dox-untreated cells (Figure 7A). These results demonstrate that the GJIC-dependent increase in intracellular ceramide production may be responsible for the improved phototoxicity of Photofrin-PDT by Cx43-mediated GJIC.
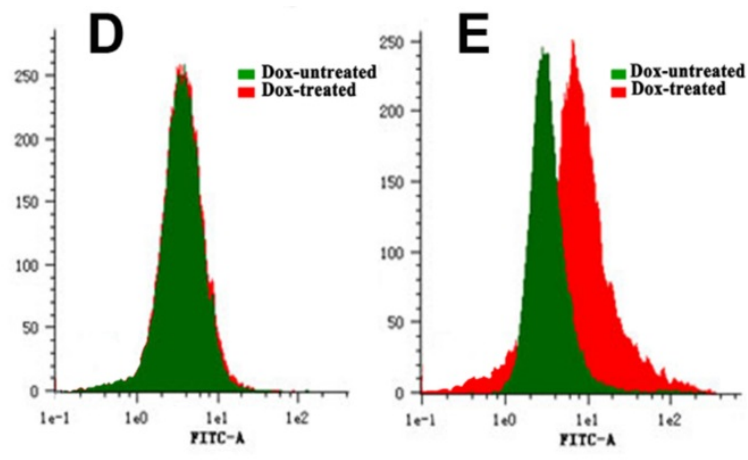

$\mathbf{F}$

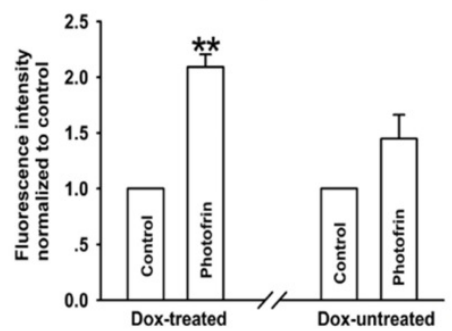

Figure 6. Enhancement of extracellular $\mathrm{Ca}^{2+}$ influx and intracellular $\mathrm{Ca}^{2+}$ release by Cx43-formed GJIC followed by PDT. The cells were pretreated with or without Dox, then incubated with Fluo-3-Am and irradiated with or without $2.5 \mathrm{mg} / \mathrm{mL}$ Photofrin. Flow cytometry was used to detect the fluorescence intensity of Ca2+ after PDT. (A): control; (B): $2.5 \mathrm{mg} / \mathrm{mL}$ Photofrin; (C): The fluorescence intensity of $\mathrm{Ca}^{2+}$. For (A), (B) and (C), the cells were incubated in fresh balanced salt solution in the absence of $\mathrm{Ca}^{2+}$ during irradiation. (D): control; $(E): 2.5 \mathrm{mg} / \mathrm{mL}$ Photofrin; $(F)$ : The fluorescence intensity of Ca ${ }^{2+}$. For $(D)$, (E) and (F), the cells were incubated in fresh balanced salt solution in the presence of $\mathrm{Ca}^{2+}$ during irradiation. Data were represented as mean \pm SD from 4 or 5 independent experiments. $t$-test was performed to analyze statistical difference between groups using SPSS 20.0. $* * P<0.01$, versus Dox-untreated group.
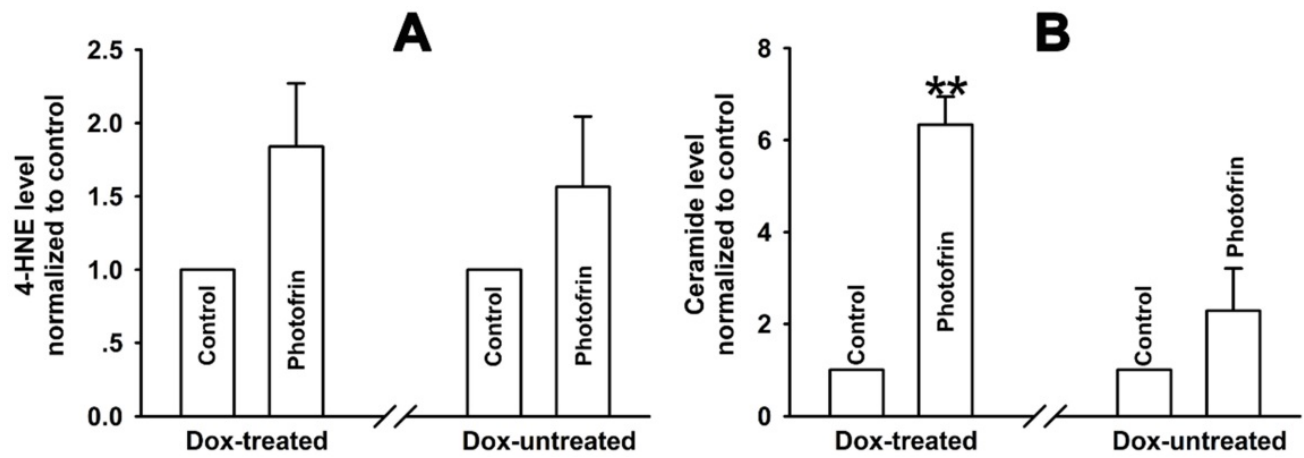

Figure 7. Elevated ceramide generation by Cx43-formed GJIC followed by PDT. The cells were pretreated with or without Dox, and then irradiated with or without $2.5 \mathrm{mg} / \mathrm{mL}$ Photofrin. The levels of 4-HNE and ceramide were detected using ELISA assay. (A): 4-HNE; (B): ceramide. For (A) and (B), data were represented as mean \pm SD from 4 and 5 independent experiments, respectively. $t$-test was performed to analyze statistical difference between groups using SPSS 20.0 . $* * P<0.01$, versus Dox-untreated group. 


\section{Discussion}

Despite it is generally believed that tumor cells have reduced connexins (Cxs) expression and/or aberrant GJIC, changes in GJIC and Cxs expression with tumorigenesis are complicated and heterogeneous. In some tumors, the expression of Cxs can be maintained or upregulated. It has been reported that $\mathrm{C} \times 43$ expression can be upregulated or downregulated in glioma cells [18]. Moreover, no changes in the expression of Cx43 is observed in small lung cancers and in larger lung malignancy [19, 20]. Since the recurrences of PDT-mediated cancer treatment of glioma and non-small cell lung cancer can occur [21, 22], Cx43-formed GJIC would be expected to play a significant role in PDT efficacy in the above cases.

The present study demonstrates that there is a substantially enhanced effect of Cx43-formed GJIC on the phototoxicity of Photofrin-PDT. At high-cell density, where cells have chances to contact with each other to form GJ, inhibiting of Cx43-formed GJIC by either pretreating with GJ inhibitor, 18a-GA, or suppressing the expression of Cx43 could significantly reduce PDT phototoxicity (Figure $2 A \sim D)$. Such an action is deficient at low density because of lacking GJ formation (Figure 1). The GJIC-dependent effects under high-cell density condition was also found in the treatment of U87 glioma cells with PDT, in which Cx43-mediated GJ inhibitors TPA and CBX could remarkably suppress PDT phototoxicity, whereas RA, a GJIC enhancer, could substantially increase the sensitivity of cells to PDT (Figure 3A I). Moreover, the effect of Cx43-mediated GJIC on PDT phototoxicity was also evaluated in vivo. The results showed that the presence of Cx43-mediated GJIC could significantly decrease the mean RTV and tumor weights in Dox-treated xenografts when compared to Dox-untreated tumors after Photofrin-PDT (Figure $4 \mathrm{~A} \sim \mathrm{D})$. The histological results showed that Cx43-composed GJIC induced by Dox resulted in a decreased density of tumor cells in the sections of xenografts after PDT (Figure 4E), indicating the ability of GJIC to improve PDT efficacy in vivo. These results indicate that upregulating $\mathrm{C} \times 43$ expression or improving the function of Cx43-composed GJIC may enhance PDT phototoxicity and suggest that treatment strategies designed to enhance the expression of $\mathrm{Cx} 43$ or to retain the function of Cx43-composed GJIC may improve PDT efficacy.

The present study makes clear that Cx43-composed GJIC may be an important influencing factor of PDT response. Thus, factors that affect the functionality of GJIC may have influences on PDT efficacy. It is now clear that several analgesics, such as tramadol and flurbiprofen, has been reported to restrain the functionality of gap junctional channels [23]. Therefore, in the event that PDT is used concurrently with these analgesics for patients who accompanies carcinous pain, PDT efficiency can be prohibited by the GJIC suppression produced by the analgesics. It has also been noted that some compounds, such as baicalein and simvastatin have been shown to augment gap junctional function, which enhances the efficiency of antineoplastic drugs by increasing toxic bystander effects [24, 25]. Thus, if PDT is used concurrently with these agents in clinical settings, the efficiency of PDT for tumors with GJIC can be increased.

It should be noted that GJIC-dependent effect on phototoxicity were disappeared at low $(1 \mu \mathrm{g} / \mathrm{mL})$ and high $(10 \mu \mathrm{g} / \mathrm{mL})$ Photofrin concentrations in the present study (Figure 2 and 3). This accounts for the fact that GJIC-dependent component of PDT efficacy relies on clinically photosensitizer' concentration used. At low concentration, the diffusion of cytotoxic substances induced by PDT via GJ channels can only kill a small portion of cells and Cx43-mediated GJIC-dependent effect would be obscured. At high concentration, more than $80 \%$ cells were directly killed and GJIC would not be expected to play a role in the end point. At the clinically appropriate Photofrin concentrations used, however, PDT phototoxicity could be augmented by gap junction-mediated intercellular diffusion of toxic products and one should note that maintaining or elevating the functionality of Cx43 GJIC may improve PDT efficacy.

It has been determined that the bystander cell killing effect of GJIC depends on the transfer of toxic substances from one cell to an adjacent other via GJ channels. Studies have shown that the generation of ROS induced by PDT leads to cellular injuries by assaulting lipids, DNA and proteins $[15,26]$, and ROS, as an oxidative stress signal, is allowed to transfer through GJ couplings [27]. Thus, intracellular ROS may account for the increased phototoxicity of Photofrin-PDT by Cx43-mediated GJIC. The results indicated that ROS generation induced by PDT in Cx43 expressing cells was substantially enhanced than that in cells not expressing $\mathrm{Cx} 43$ (Figure 5A C). The above results demonstrate that the intercellular ROS diffusion may be responsible for the enhanced phototoxic action of PDT by Cx43-mediated GJIC.

It has been established that the enhanced $\left[\mathrm{Ca}^{2+}\right]_{\mathrm{i}}$ owing to the increased $\mathrm{Ca}^{2+}$ influx and release induced by PDT can cause cell death and apoptosis $[28,29]$ and $\mathrm{Ca}^{2+}$ can transfer from one cell to surrounding unexposed other via GJ couplings [30]. Therefore, the increased PDT cytotoxicity modulated 
by Cx43-formed GJIC may arise from the intracellular $\mathrm{Ca}^{2+}$ diffusion. However, studies have shown that the rise of $\left[\mathrm{Ca}^{2+}\right]_{i}$ may close GJ complexes [31]. It should be noted that the effective level of intracellular $\mathrm{Ca}^{2+}$ on GJ couplings rests upon the species of cell and Cx expressed [32]. The data that the level of intracellular $\mathrm{Ca}^{2+}$ was increased by Cx43-composed GJIC indicated that PDT-induced increase in $\left[\mathrm{Ca}^{2+}\right]_{\mathrm{i}}$ had no effects on the channels composed by $\mathrm{C} \times 43$ (Figure 6A F). Notably, the results demonstrate that Cx43-mediated GJIC has the ability to facilitate $\mathrm{Ca}^{2+}$ transfer, which may account for the raised phototoxicity of Photofrin-PDT by Cx43-composed GJIC (Figure $6 \mathrm{~A} \sim \mathrm{F})$.

Reports have shown that the improved lipid peroxides generation is responsible for apoptosis and cell death induced by PDT [10, 11, 33]. Among these lipid peroxides, 4-HNE and ceramide may transfer through GJ complexes, causing the rise of phototoxicity owing to their small molecular weights $(<1.5 \mathrm{kDa})$. Thus, theoretically, the improvement of PDT phototoxicity dependent on Cx43-mediated GJIC may be associated with this mechanism. The results showed that Cx43-mediated GJIC was able to elevate the intracellular generation of ceramide after PDT, whereas the presence of GJIC could not alter the intracellular level of 4-HNE after PDT (Figure 7A and $B)$, indicating that the increased phototoxic effect of GJIC mediated by Cx43 may be related with ceramide-mediated lipid peroxide pathway.

Taken together, the present study presents the enhanced effects of Cx43-composed GJIC on PDT-mediated cancer treatment and indcates that the function of $\mathrm{C} \times 43$-mediated gap junctional couplings in malignant tumors may be an important decisive factor of the response to PDT in clinic. Thus, several beneficial therapeutic strategies should be considered. Firstly, retaining or even transitorily elevating the expression of $\mathrm{Cx} 43$ and the function of Cx43-composed GJIC can enhance PDT phototoxicity, resulting in a remarkable increase in therapeutic effect of PDT. Oppositely, factors disturbing Cx43-composed GIIC may cause the reduction of malignancies' sensitivity to PDT-mediated cancer treatment, leading to a substantial decrease in PDT efficacy.

\section{Acknowledgments}

This project was supported by the Natural Science Foundation of China (No. 81402946), China Postdoctoral Science Foundation (No. 2016M591927), the Natural Science Research grant of Higher Education of Jiangsu province, China (No.14KJD310002) and Initializing Fund of Xuzhou
Medical University, China (No. D2014010 and D2014017).

\section{Competing Interests}

We declare that all the authors listed do not have any competing financial interests.

\section{References}

1. Shafirstein G, Bellnier D, Oakley E, Hamilton S, Potasek M, Beeson K, et al. Interstitial Photodynamic Therapy-A Focused Review. Cancers. 2017; 9.

2. Buchholz J, Walt H. Veterinary photodynamic therapy: A review. Photodiagn Photodyn. 2013; 10: 342-7.

3. Pazurek M, Malecka-Panas E. Photodynamic therapy in the palliative treatment of esophageal cancer. Photodiagn Photodyn. 2005; 2: 73-7.

4. Abbade L, Gige T, Amaral V, Aquino S, Miot H. Higher recurrence rates of head and neck nodular basal cell carcinoma treated with topical photodynamic therapy (MAL-PDT) compared to surgical excision: A randomized controlled study. J Am Acad Dermatol. 2015; 72: Ab185-Ab.

5. Aasen T, Mesnil M, Naus CC, Lampe PD, Laird DW. Gap junctions and cancer: communicating for 50 years. Nature reviews Cancer. 2017; 17: 74.

6. Hong XT, Wang Q, Yang Y, Zheng SP, Tong XH, Zhang SZ, et al. Gap junctions propagate opposite effects in normal and tumor testicular cells in response to cisplatin. Cancer Lett. 2012; 317: 165-71.

7. Autsavapromporn N, Suzuki M, Funayama T, Usami N, Plante I, Yokota Y, et al. Gap Junction Communication and the Propagation of Bystander Effects Induced by Microbeam Irradiation in Human Fibroblast Cultures: The Impact of Radiation Quality. Radiat Res. 2013; 180: 367-75.

8. Wang SQ, Zhang SW, Zhang CZ, Zhao ZY, Wang YJ. Connexin 43 enhances oxaliplatin cytotoxicity in colorectal cancer cell lines. Cell Mol Biol. 2017; 63: 53-8.

9. Marconi P, Tamura M, Moriuchi S, Krisky DM, Niranjan A, Goins WF, et al. Connexin 43-enhanced suicide gene therapy using herpesviral vectors. Molecular therapy : the journal of the American Society of Gene Therapy. 2000; 1: 71-81.

10. Chiu SM, Davis TW, Meyers M, Ahmad N, Mukhtar H, Separovic D. Phthalocyanine 4-photodynamic therapy induces ceramide generation and apoptosis in acid sphingomyelinase-deficient mouse embryonic fibroblasts. Int J Oncol. 2000; 16: 423-7.

11. Girotti AW, Kriska T. Role of lipid hydroperoxides in photo-oxidative stress signaling. Antioxid Redox Sign. 2004; 6: 301-10.

12. Wu DP, Fan LX, Xu CF, Liu Z, Zhang Y, Liu L, et al. GJIC Enhances the phototoxicity of photofrin-mediated photodynamic treatment by the mechanisms related with ROS and Calcium pathways. J Biophotonics. 2015; 8: 765-74.

13. Wu DP, Lin TY, Bai LR, Huang JL, Zhou Y, Zhou N, et al. Enhanced Phototoxicity of Photodynamic Treatment by Cx26-composed GJIC via ROS-, calcium- and lipid peroxide-mediated pathways. J Biophotonics. 2017.

14. Wu DP, Liu Z, Fu YN, Zhang $\mathrm{Y}$, Tang $N$, Wang $\mathrm{Q}$, et al. Efficacy of 2-(1-hexyloxyethyl)-2-devinyl pyropheophorbide-a in photodynamic therapy of human esophageal squamous cancer cells. Oncol Lett. 2013; 6: 1111-9.

15. Rajendran M. Quinones as photosensitizer for photodynamic therapy: ROS generation, mechanism and detection methods. Photodiagn Photodyn. 2016; 13: $175-87$

16. Cali B, Ceolin S, Ceriani F, Bortolozzi M, Agnellini AHR, Zorzi V, et al. Critical role of gap junction communication, calcium and nitric oxide signaling in bystander responses to focal photodynamic injury. Oncotarget. 2015; 6: 10161-74.

17. Saez JC, Schalper KA, Retamal MA, Orellana JA, Shoji KF, Bennett MVL. Cell membrane permeabilization via connexin hemichannels in living and dying cells. Exp Cell Res. 2010; 316: 2377-89.

18. Sin WC, Crespin S, Mesnil M. Opposing roles of connexin43 in glioma progression. Biochimica et biophysica acta. 2012; 1818: 2058-67.

19. Udaka N, Miyagi $Y$, Ito T. Connexin expression in mouse lung tumor. Cancer Lett. 2007; 246: 224-9.

20. Isakson BE, Seedorf GI, Lubman RL, Evans WH, Boitano S. Cell-cell communication in heterocellular cultures of alveolar epithelial cells. Am J Resp Cell Mol. 2003; 29: 552-61.

21. Akopov A, Rusanov A, Gerasin A, Kazakov N, Urtenova M, Chistyakov I. Preoperative endobronchial photodynamic therapy improves resectability in initially irresectable (inoperable) locally advanced non small cell lung cancer. Photodiagnosis Photodyn Ther. 2014; 11: 259-64.

22. Zavadskaya capital Te C. Photodynamic therapy in the treatment of glioma. Experimental oncology. 2015; 37: 234-41.

23. He B, Tong X, Wang L, Wang Q, Ye H, Liu B, et al. Tramadol and flurbiprofen depress the cytotoxicity of cisplatin via their effects on gap junctions. Clinical cancer research : an official journal of the American Association for Cancer Research. 2009; 15: 5803-10

24. Wang LZ, Peng JX, Huang HS, Wang Q, Yu ML, Tao L. Simvastatin protects Sertoli cells against cisplatin cytotoxicity through enhanced gap junction intercellular communication. Oncol Rep. 2015; 34: 2133-41. 
25. Wang YW, Wang Q, Zhang SZ, Zhang Y, Tao L. Baicalein increases the cytotoxicity of cisplatin by enhancing gap junction intercellular communication. Mol Med Rep. 2014; 10: 515-21.

26. Wallat JD, Wek KS, Chariou PL, Carpenter BL, Ghiladi RA, Steinmetz NF, et al. Fluorinated polymer-photosensitizer conjugates enable improved generation of ROS for anticancer photodynamic therapy. Polym Chem-Uk. 2017; 8: 3195-202.

27. Brady NR, Elmore SP, van Beek JJHGM, Krab K, Courtoy PJ, Hue L, et al. Coordinated behavior of mitochondria in both space and time: A reactive oxygen species-activated wave of mitochondrial depolarization. Biophys J. 2004; 87: 2022-34.

28. Song L, Li CZ, Zou Y, Dai F, Luo XQ, Wang BK, et al. O-2 and Ca2+ Fluxes as Indicators of Apoptosis Induced by Rose Bengal-Mediated Photodynamic Therapy in Human Oral Squamous Carcinoma Cells. Photomed Laser Surg. 2015; 33: 258-65.

29. Hong $X$, Jiang F, Kalkanis SN, Zhang ZG, Zhang XP, Zheng XG, et al. Intracellular free calcium mediates glioma cell detachment and cytotoxicity after photodynamic therapy. Laser Med Sci. 2009; 24: 777-86.

30. Goldberg GS, Moreno AP, Lampe PD. Selective transfer of endogenous metabolites through gap junctions formed by specific connexins. Mol Biol Cell. 2002; 13: 352a-a.

31. Peracchia C. Chemical gating of gap junction channels Roles of calcium, $\mathrm{pH}$ and, calmodulin. Bba-Biomembranes. 2004; 1662: 61-80.

32. Lazrak A, Peres A, Giovannardi S, Peracchia C. Ca-Mediated and Independent Effects of Arachidonic-Acid on Gap-Junctions and Ca-Independent Effects of Oleic-Acid and Halothane. Biophys J. 1994; 67: 1052-9.

33. Benov L. Photodynamic Therapy: Current Status and Future Directions. Med Prin Pract. 2015; 24: 14-28. 